Abstract E-063 Table 1 Comparing accuracy of the individual scales performed in this trial

\begin{tabular}{|c|c|c|c|c|c|c|c|c|c|}
\hline StrokeSeverity Scale & Score Range & Optimal Score Cut Point (CP) & Overall Accuracy & Sensitivity & Specificity & Area Under the Curve & PPV & NPV & Odds Ratio \\
\hline PREDICT-3 & $0-3$ & $\geq 3$ & 0.75 & 0.45 & 0.80 & $0.65,95 \% \mathrm{Cl}[0.55-0.75]$ & 0.26 & 0.90 & $1.72,95 \% \mathrm{Cl}[1.17,2.53]$ \\
\hline PASS & $0-3$ & $\geq 2$ & 0.61 & 0.74 & 0.59 & $0.67,95 \% \mathrm{Cl}[0.57,0.77]$ & 0.22 & 0.94 & $1.93,95 \% \mathrm{Cl}[1.26,3.0]$ \\
\hline PREDICT-4 & $0-4$ & $\geq 3$ & 0.67 & 0.55 & 0.69 & $0.64,95 \% \mathrm{Cl}[0.54,0.75]$ & 0.21 & 0.91 & $1.51,95 \% \mathrm{Cl}[1.10,2.05]$ \\
\hline RACE & $0-9$ & $\geq 6$ & 0.79 & 0.74 & 0.80 & $0.80,95 \% \mathrm{Cl}[0.72,0.88]$ & 0.37 & 0.95 & $1.57,95 \% \mathrm{Cl}[1.32,1.87]$ \\
\hline RACE (at traditional CP) & $0-9$ & $\geq 5$ & 0.74 & 0.77 & 0.74 & $0.80,95 \% \mathrm{Cl}[0.72,0.88]$ & 0.31 & 0.96 & $1.57,95 \% \mathrm{Cl}[1.32,1.87]$ \\
\hline
\end{tabular}

portion, or proximal aspect of single or multiple M2 branches of MCA, or basilar artery (BA). We used SAS and c-statistics to create receiver-operating characteristic (ROC) curves to determine the area under the curve (AUC) and optimal score cut point (CP) for each scale. We also calculated the sensitivity, specificity, positive predictive value (PPV), negative predictive value (NPV) and overall accuracy for each optimal CP score.

Results 250 subjects were enrolled, 17 were excluded for lack of neurovascular imaging, 1 was excluded for incomplete stroke scale data, leaving 232 subjects who met inclusion criteria for analysis. 31 subjects had LVO and the prevalence of LVO was $13.4 \%$. In table 1 , we report the performance of each stroke severity scale at their respective optimal CP. In our study, the optimal CP for RACE was $\geq 6$ while the original RACE study was $\geq 5$.

Conclusions This large, US-based, prospective, pre-hospital, EMS-administered study showed greater AUC for RACE. However, all three scales were non-inferior in AUC with respect to RACE. These newer, simpler scales may have acceptable diagnostic ability for LVO and be more easily adopted by EMS.

Disclosures R. James: 1; C; Medtronic, Medtronic. A. Cruz: None. E. Fortuny: None. B. Ugiliweneza: None. D. Wang: None. A. White: None. N. Khattar: None. S. Adams: None. B. Gallinore: None. D. Ding: None. S. Wolfe: None. D. Heck: None.

\section{E-064 DIFFERENTIAL RESPONSE OF ATRIAL FIBRILLATION ASSOCIATED STROKE WITH THROMBOLYSIS AND MECHANICAL THROMBECTOMY}

${ }^{1} \mathrm{~F}$ Akbik*, ${ }^{2} \mathrm{~A}$ Alawieh, ${ }^{2} \mathrm{C}$ Cawley, ${ }^{2} \mathrm{~B}$ Howard, ${ }^{2} \mathrm{~F}$ Tong, ${ }^{3} \mathrm{~F}$ Nahab, ${ }^{1} \mathrm{O}$ Samuels, ${ }^{4} \mathrm{I}$ Maier, ${ }^{5} \mathrm{~W}$ Feng, ${ }^{6} \mathrm{~N}$ Goyal, ${ }^{7} \mathrm{R}$ Starke, ${ }^{8} \mathrm{~A}$ Rai, ${ }^{9} \mathrm{~K}$ Fargen, ${ }^{10} \mathrm{M}$ Anadani, ${ }^{11} \mathrm{M}$ Psychogios, ${ }^{12} \mathrm{R}$ De Leacy, ${ }^{10}$ S Keyrouz, ${ }^{13} \mathrm{~T}$ Dumont, ${ }^{14 \mathrm{P}}$ Kan, ${ }^{15} \mathrm{~J}$ Lena, ${ }^{16} \mathrm{~J}$ Liman, ${ }^{17} \mathrm{~A}$ Arthur, ${ }^{18} \mathrm{~L}$ Elijovich, ${ }^{19} \mathrm{D}$ Mccarthy, ${ }^{20} \mathrm{~V}$ Saini, ${ }^{21} \mathrm{~S}$ Wolfe, ${ }^{12} \mathrm{~J}$ Mocco, ${ }^{12} \mathrm{JT}$ Fifi, ${ }^{22} \mathrm{~F}$ Nascimento, ${ }^{10} \mathrm{~J}$ Giles, ${ }^{23} \mathrm{R}$ Crosa, ${ }^{24} \mathrm{~W}$ Fox, ${ }^{25} \mathrm{~B}$ Gory, ${ }^{15} \mathrm{~A}$ Spiotta, ${ }^{2} \mathrm{~J}$ Grossberg. ${ }^{1}$ Neurology, Neurosurgeny, Emory University, Atlanta, GA; ${ }^{2}$ Neurosurgery, Emory University, Atlanta, GA; ${ }^{3}$ Neurology, Emory University, Atlanta, GA; ${ }^{4}$ Neurology, Universitätsmedizin Göttingen, Göttingen, GERMANY; ${ }^{5}$ Neurology, Duke University, Durham, NC; ${ }^{6}$ Neurology, UT Memphis, Memphis, TN; ${ }^{7}$ Neurosurgery, University of Miami, Miami, FL; ${ }^{8}$ Radiology, West Virginia University, Morgantown, WV; ${ }^{9}$ Wake Forest Health, Winston-Salem, NC; ${ }^{10}$ Neurology, Washington University in St. Louis, St. Louis, MO; ${ }^{11}$ Neuroradiology, University Medical Center Göttingen, Göttingen, GERMANY; ${ }^{12}$ Neurosurgeny, Mount Sinai Hospital, New York, NY; ${ }^{13}$ Neurosurgery, Banner University of Arizona Medical Center, Tucson, AZ; ${ }^{14}$ Neurosurgery, Baylor College of Medicine, Houston, TX; ${ }^{15}$ Neurosurgery, Medical University of South Carolina, Charleston, SC; ${ }^{16}$ Neurology, University Medical Center Göttingen, Göttingen, GERMANY; ${ }^{17}$ Neurosurgery, Semmes Murphy, Memphis, TN; ${ }^{18}$ Neurology, Semmes Murphy, Memphis, TN: ${ }^{19}$ Neurology, Neurosurgery, Boston University Medical Center, Boston, MA; ${ }^{20}$ Neurology, University of Miami, Miami, FL; ${ }^{21}$ Neurosurgery, Wake Forest Health, Winston-Salem, NC; ${ }^{22}$ Neurology, Baylor College of Medicine, Houston, TX; ${ }^{23}$ Neurology, Médica Uruguaya, Montevideo, URUGUAY; ${ }^{24}$ Neurosurgery, University of Michigan, Ann Arbor, Mli ${ }^{25}$ University Hospital of Nancy, Nancy, FRANCE
Introduction Atrial fibrillation (AF) associated stroke is associated with worse functional outcomes, less effective recanalization, and increased rates of hemorrhagic complications after intravenous thrombolysis (IVT). It remains unclear whether recanalization efficacy, procedural speed, and hemorrhagic complications differ in AF associated stroke treated with mechanical thrombectomy (MT).

Methods In a retrospective multicenter study of 4,232 patients who underwent MT, 3,385 patients had anterior circulation large vessel occlusions (LVO). 1,210 (35.7\%) patients had comorbid AF, diagnosed either before or on presentation. Baseline characteristics, procedural outcomes, and clinical outcomes were reported and compared.

Results AF was associated with faster procedural time (51.5 vs. 58.2 minutes, $p=0.007)$, higher rates of first pass success $(42 \%$ vs. $35 \%, \mathrm{p}=0.001)$, and comparable angiographic outcomes. In multivariate analysis, AF was an independent predictor of both procedural speed and first pass success. AF patients had worse functional outcomes, attributable to increased age and stroke severity at presentation. In contrast to IVT associated sICH in AF patients, there was no additive risk of sICH after MT (aOR 0.95, 0.65-1.38, p=0.791). When patients who received IVT-MT were dichotomized by reperfusion status, only patients with poor reperfusion trended towards increased rates of $\mathrm{sICH} \quad(\mathrm{aOR}=2.69,0.96-7.53$, $\mathrm{p}=0.06)$.

Conclusions MT in AF patients is associated with increased rates of rapid reperfusion without added risk of sICH when reperfusion is achieved. Even when combined with IVT, MT in AF patients does not carry an added risk of sICH if successful recanalization is achieved. Given the historically low recanalization efficacy of IVT for AF associated stroke, it is unclear whether IVT is additive in the setting of AF associated LVO undergoing MT, particularly given the trend towards increased sICH if combined IVTMT therapy ends with poor reperfusion. Randomized studies are warranted to evaluate whether AF patients with acute LVO may represent a subgroup of patients who may benefit from MT alone versus combined IVT-MT in thrombectomy capable centers.

Disclosures F. Akbik: None. A. Alawieh: None. C. Cawley: None. B. Howard: None. F. Tong: None. F. Nahab: None. O. Samuels: None. I. Maier: None. W. Feng: None. N. Goyal: None. R. Starke: None. A. Rai: None. K. Fargen: None. M. Anadani: None. M. Psychogios: None. R. De Leacy: None. S. Keyrouz: None. T. Dumont: None. P. Kan: None. J. Lena: None. J. Liman: None. A. Arthur: None. L. Elijovich: None. D. Mccarthy: None. V. Saini: None. S. Wolfe: None. J. Mocco: None. J. T Fifi: None. F. Nascimento: None. J. Giles: None. R. Crosa: None. W. Fox: None. B. Gory: None. A. Spiotta: None. J. Grossberg: None. 